\title{
Development of Operation System for Electric Wheelchair by Voluntary Movements of Head
}

\author{
Taisei Kusakabe, Kohei Watanabe, Shota Matsuhashi, Ryoji Onodera and Michiaki Shishido* \\ National Institute of Technology, Tsuruoka College, 104 Sawada Inooka, Tsuruoka Yamagata 997-8511, Japan \\ *Corresponding Author: m-shishido@tsuruoka-nct.ac.jp
}

\begin{abstract}
Recent almost developed countries like Japan, senior people are increasing. In such as aging society, supplying human resource to care the senior people doesn't catch up with its demands. Therefore, the societies encourage the cared elderly people become to manage to live by themselves in their life. To realize such as the society of independent elderly people, many assistive machines and tools are developed. In particular, electric wheelchairs are very effective to keep and improve their Quality of Life (QOL). In this study, to promote the use of electric wheelchairs, a novel operation system for electric wheelchair using pointing device (Hands-Free Operation-Interface) is proposed. The proposed system can operate an electric wheelchair without body action. To investigate operation system of the wheelchair, its driving performance is compared with driving using a standard joystick under same slalom course running. There were some obstacles in the slalom course, driver needed to drive the electric wheelchair and make own way around these obstacles to goal. In this experiment, successratio and running time are compared between Hands-Free Operation-Interface and standard joystick operation to evaluate the operability of the systems. As a result, it is suggested that success-ratio is depended on operators, and the average of success-ratio is $67 \%$. Also running time is observed that depended on driving ability of operators. Therefore, these results showed that practice and adapting of users are necessary to drive the electric wheelchair using the proposed operation system. In addition, this study showed some direction for controlling the system safely.
\end{abstract}

Keywords: Electric wheelchair, Movements of head, Novel operation system, Welfare equipment.

\section{Introduction}

Recent years, gap between human resource and demands on nursing-care is wider with low-birth rate and high aging speed in Japanese society. Thus, workloads of care-workers are increasing. In such background, current society need to encourage independence of care-receivers including elderly people in their life. Nowadays, to realize such as independent society in care-receivers, many assistive machines and tools are under development for reducing careworkers. Especially, electric wheelchair is expected to keep or improve care-receivers' $\mathrm{QOL}^{(1)}$.

On the other hand, conventional electric wheelchairs need to be controlled and handled by body actions of the user. Thus, patients who have handicaps or limit on their upper limbs of their body, it is difficult to drive the electric wheelchair for such as patients. Then, head of the patients are candidate of control-center as previous report ${ }^{(2)}$.

The authors developed and evaluated a Hands-Free Operation-Interface. The interface was evaluated pointing performance based on ISO9241-9(3) . The Hands-Free Operation-Interface detects electric potential of masseter muscle and head position. By measuring electric potential of the muscle, it is determined whether occlusion of mouth as voluntary action or not. This electric signal from the muscle can be applied for some signals as "stop" and "go" the electric wheelchair. In addition, angle of head position can detect the face orientation of the wheelchair user. Human have a characteristic that they turn their face to the direction before launching their action ${ }^{(4,5)}$. Thus, it is expected to realize a novel operation system which be intuitive controlled by corresponding between detected face orientation and the progressing direction of the wheelchair.

In this study, a novel operation system of electric 


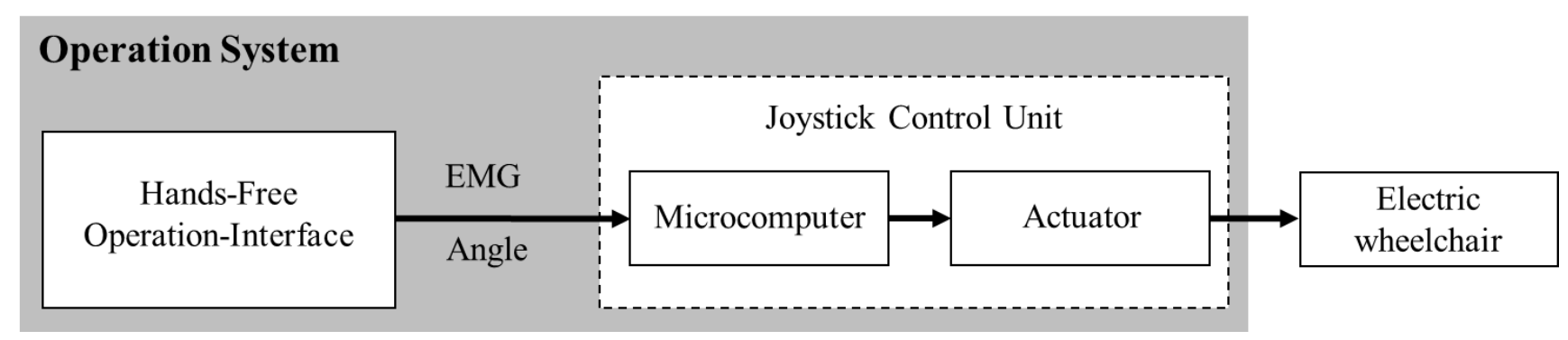

Fig. 1. Schematic diagram of the system.

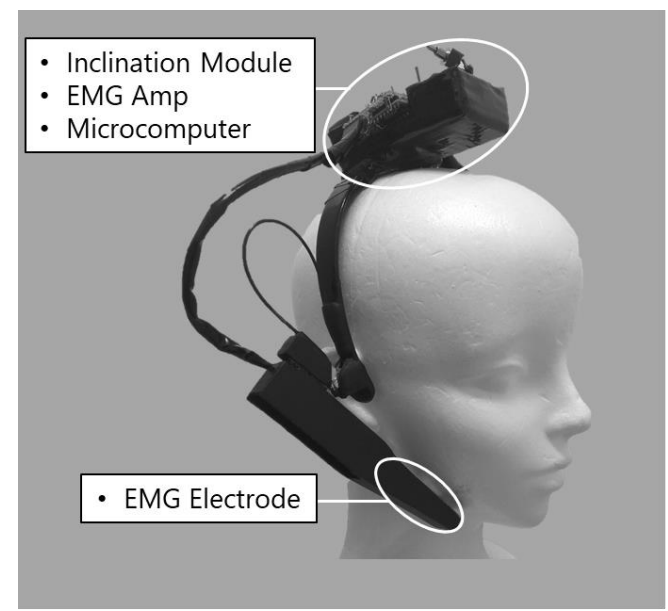

Fig. 2. Overview of

Hands-Free Operation-Interface.

wheelchair using Hands-Free Operation-Interface without controlling from upper limbs of human body is proposed. To evaluate operability of this developed system, it is recorded success-ratio and the running time on a slalom running.

\section{System overview}

Figure 1 shows the schematic diagram of the operation system. The system is fabricated from the Hands-Free Operation- Interface and a unit for controlling a joystick (Joystick Control Unit) corresponding to the interface.

Figure 2 shows the overview of Hands-Free OperationInterface. The interface is assembled by some parts; an inclination module (MPU-6050, InvenSense) equipped with three-axis acceleration sensor and three-axis gyro sensor, a microcomputer (Arduino Pro Mini, Arduino), and noninvasive dry electrodes for electromyography (EMG). The total weight of the Hands-Free Operation-Interface is $230 \mathrm{~g}$. The inclination module which is placed at the top of the head detected angular velocity of head. The data is converted into the value of the yaw angle of head by the microcomputer. At the same time, the myoelectric potentials generated by occlusion are detected from the electrodes placed in the left and right masseter muscles ${ }^{(3)}$. These myoelectric potentials are converted to a digital signal by the microcomputer. When occlusion is not detected, this digital signal is designated as 0 and occlusion is detected, it is designated as 1 respectively.

Figure 3. shows the overview of Joystick Control Unit. The unit is designed on the premise that it is attached to a standard joystick type electric wheelchair. This unit consists of an inclination module (MPU-6050, InvenSense) same to Hands-Free Operation-Interface, a microcomputer (Arduino Uno, Arduino), two actuators (MG996R, TowerPro), and joystick control mechanism elements. The weights of the microcomputer and the inclination module, batteries for actuators, and actuators and mechanism elements are $120 \mathrm{~g}$, $340 \mathrm{~g}$, and $170 \mathrm{~g}$ respectively. To each actuator, four AA batteries are installed as a $6.0 \mathrm{~V}$ DC power supply. Also, the value of the yaw angle of the electric wheelchair is calculated from the angular velocity collected by the inclination module of this unit.

Figure 4. shows the image of mechanical parts for controlling the joystick. The joystick has a control unit. The joystick is included as a mechanical component in the system, also the command of joystick is inputted by the controlling actuators. 
Hands-Free Operation-Interface transmits a digital signal to Joystick Control Unit at all time. Then, with the rise at which the digital signal transitions from 0 to 1 as a signal, the serial communication between the devices and the operation of the electric wheelchair are started or stopped. The operation system is started by this digital signal. Processing-flow during the operation is described as below.

1) Joystick Control Unit requests data transmission to Hands-Free Operation-Interface.

2) Hands-Free Operation-Interface which received the transmission request, it transmits the value of the yaw angle of the head to Joystick Control Unit.

3) Joystick Control Unit received the value of yaw angle of the head detects the orientation of operator's face from the difference between the yaw angle of the head and electric wheelchair.

4) The progressing direction of the electric wheelchair is determined via the detected face direction, and the actuators control the joystick.

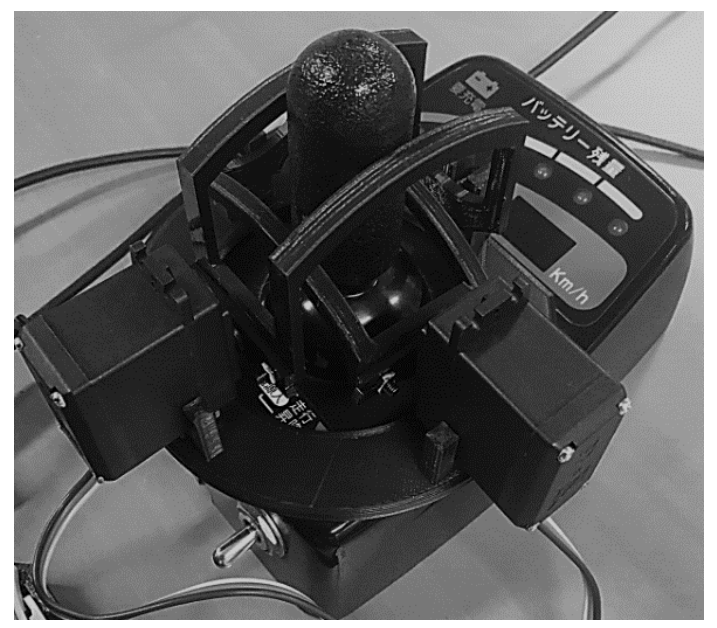

Fig. 4. Image of mechanical parts for controlling the joystick.

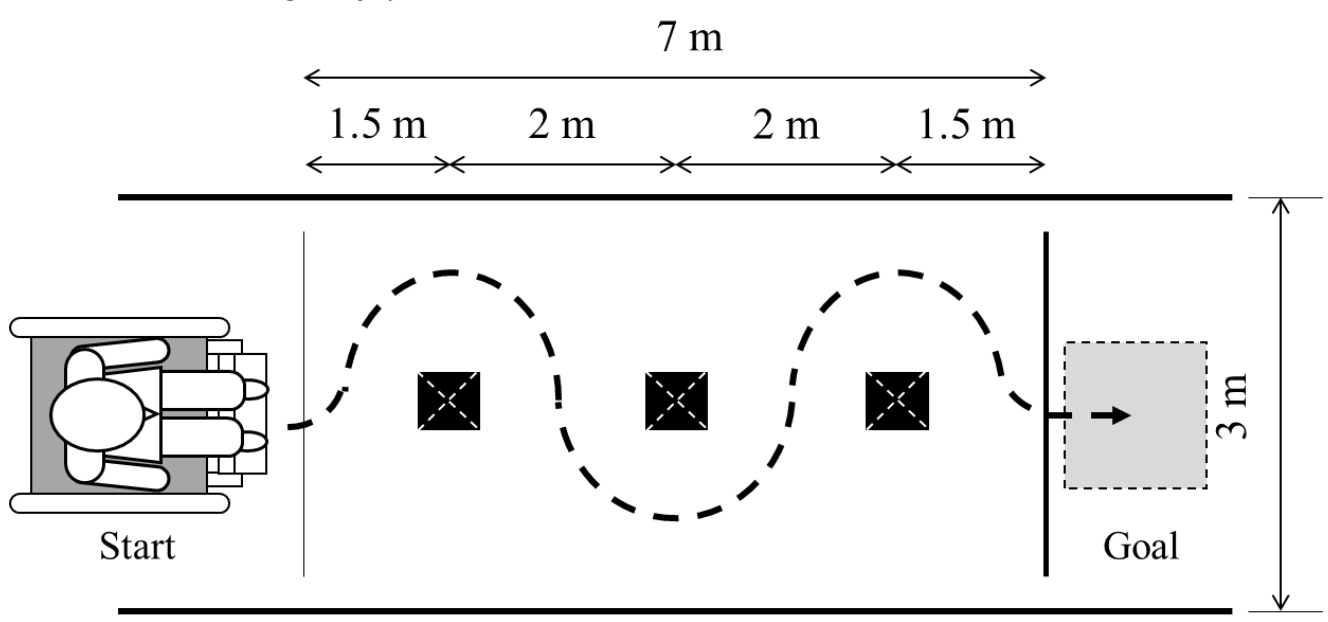

Fig. 5. Outline of the experiment condition.
The processes from 1 to 4 are repeated until the rising edge of the digital signal is detected again.

\section{Experimental}

In order to evaluate the operability of the electric wheelchair by the developed operation system, the successratio and the running time to finish the slalom course were recorded. There are a few obstacles on the course. In this experiment, the "success" in the test-running is defined what is completed all conditional below.

- The electric wheelchair did not go off the course.

- The electric wheelchair did not touch the obstacles.

- During the running, the electric wheelchair did not stop more than 10 seconds with non-intended accident by the operator.

- During the running, the electric wheelchair did not need emergency stop with shutting down of the main power supply.

Figure 5. shows the outline of this experiment condition. IMASEN EMC-240 electric wheelchair was used in this experiment. The maximum speed of this electric wheelchair needs to be chosen from $2.5,4.5$, or $6.0 \mathrm{~km} / \mathrm{h}$ on the specification of the electric wheelchair. On the other hand, The Real Estate Fair Trade Council of Federation has defined the "required walking time" to $80 \mathrm{~m} / \mathrm{min}$ (i.e. $4.8 \mathrm{~km} / \mathrm{h}$ ) ${ }^{(6)}$. Therefore, $4.5 \mathrm{~km} / \mathrm{h}$ is the closest ruled speed so that it is determined as the maximum speed of the electric wheelchair in this experiment. The three obstacles placed on the course were road-cones with a square bottom (38 $\mathrm{cm}$ each side). Participants were 6 non-handicapped men whose average ages is 20 years old. In addition, to compare with Hands-Free 


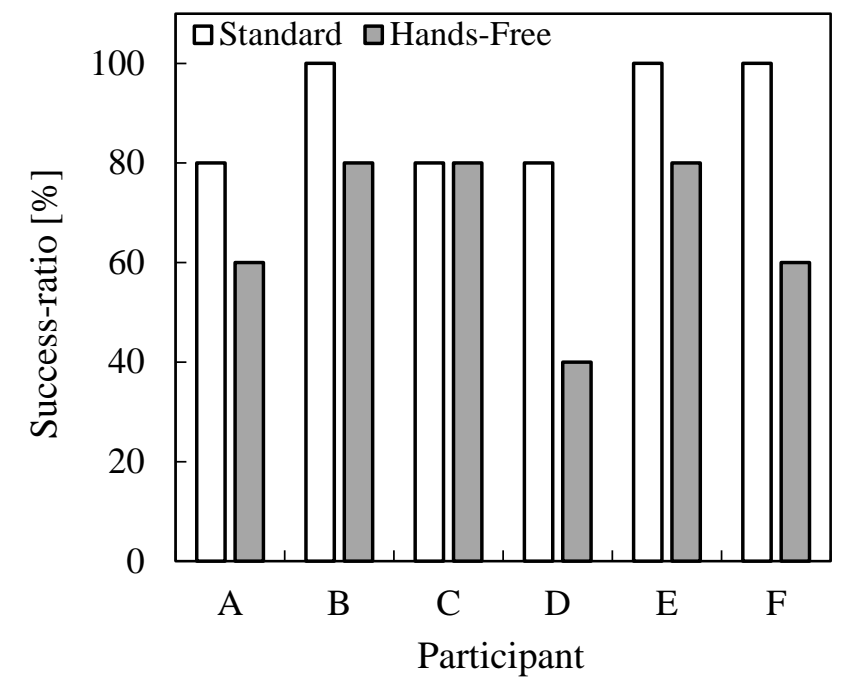

(a) Success-ratio by each operation method.

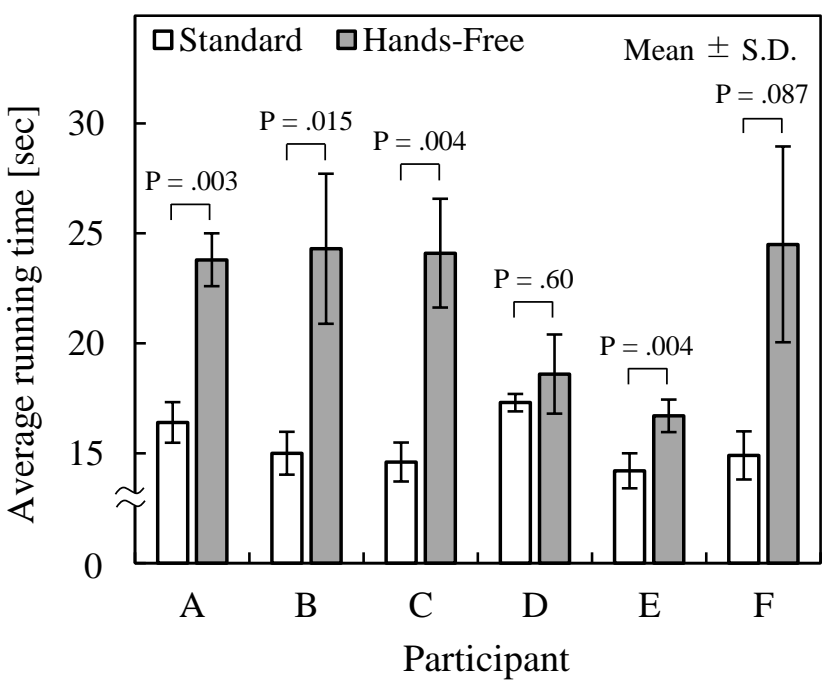

(b) Average running time by each operation method.

Fig. 6. Success-ratio and average running times for each participant.

Operation-Interface, the participants drove the electric wheelchair using standard joystick. Each participant practiced both of the hands-free operation and standard operation method, after that they tried test-running by each operation in each five times.

The significant difference of running times were evaluated by two-sided Welch's $t$-test. In these tests, a P value less than 0.05 was considered statistically significant.

\section{Results}

Hereafter, standard joystick operation and developed operation system are defined "Standard" and "Hands-Free" respectively.

Figure 6. (a) shows the results in average of successratio, "Standard" and "Hands-Free" are 90\% and 67\% respectively. In addition, success-ratio of "Standard" are higher than its "Hands-Free" in all participants, even lowest participant achieved high success-ratio, $80 \%$ in "Standard" . On the other hand, in "Hands-Free", difference of success ratio between maximum and minimum is $40 \%$. This result suggests results in "Hands-Free" is depended on operability of each participant.

Figure 6. (b) shows results in the average running times of each method. These results in running times are used only "success". The average running time of "Standard" is shorter than "Hands-Free" in all participants. Especially, in participant A, B, C, E, it is observed a significant deference between "Standard" and "Hands-Free" $(\mathrm{P}<.05)$. The average running time of "Standard" and "Hands-Free" are $15.4 \mathrm{sec}$ and $22.0 \mathrm{sec}$ respectively.

\section{Discussion}

The failure (not success) of operating wheelchair in this experiment, its $85 \%$ was collision onto the obstacles. Moreover, over its $60 \%$ was rear wheel touching on the obstacle. This result suggests that it is difficult for operators to view the rear of wheelchair. The difficulty of rearview is suggested from that the system for monitoring around the backwards of wheelchair has developed as previous $\operatorname{research}^{(7)}$. Especially, in "Hands-Free", this tendency is observed more frequent than its "Standard". Because the participants were required to operate the wheelchair using controlling their face orientation in "Hands-Free" method. Toward future work, it is necessary to develop another system which is able to check the rearview with keeping face orientation. Also almost participants drove excessive route not to touch the obstacle so that running time of "Hands-Free" is longer than its "Standard".

In both results of success-ratio and running time in "Hands-Free" and "Standard", "Standard" is more stable and less dependent on participants than "Hands-Free". On the other hand, it is observed that "Hands-Free" depends highly on ability of participants. Thus, stability of "HandsFree" operation is improved by practice and adaption of operator. Now, adaptation is defined the state that the operator is able to operate the wheelchair intentionally. This adaptation is depended on personal ability and character. Therefore, even practice times of all participants is same, the distribution of results in "Hands-Free" is highly.

Moreover, it is cleared the direction toward future 
work in this research. That is necessary of improving hardware of control system. To operate correctly the system by voluntary movements of operator, the Hands-Free Operation-Interface is necessary to be worn the operator stably. However, sometimes wearing of the interface was difficult for unsuited head shape of participants. By changing the design of this interface, it might be improved. In addition, in the case of operating the system on long time, the detection of the face orientation is observed error. So that it was necessary to modify the operation system when the wheelchair didn't move correctly to operator's intended direction. To promote independence of users in their life, this error detecting needs to be minimum.

In these experimental results, improvement points of this system were brought clarity. According to modify these points, operability, safety and maintainability are improved, and it is expected that it will be a more useful system.

\section{Conclusion}

In this study, a novel operation system of electric wheelchair without the limbs of human body action is developed. Success-ratio and the running time on a slalom running were recorded so that the operability of this system was investigated. The summary of the obtained results was shown as follows.

1) "Hands-Free" operation is depended on personal operability of operators rather than its "Standard".

2) In "Hands-Free" operation, it is difficult for operators to check the backwards. In addition, over rounding around the obstacles to avoid them made operators take longer running time in "Hands-Free" operation.

3) For realizing our proposed system, it is necessary to improve the hardware design and to modify detecting face orientation systems.

\section{References}

(1) Japanese Association of Occupational Therapists :

"Guide Book of Leaving-off from bed for Patients; To

Promote Their Actions and Participation in Practical method (Practical method version) " [in Japanese], http://www.jaot.or.jp/wp-

content/uploads/2011/04/guide-jissen.pdf (accessed

2018-11-16)

(2) Nobuaki Nakazawa : "Human Interface for Welfare Equipment" [in Japanese], Journal of the Japan Society of Applied Electromagnetics, Vol. 25, No. 3, pp. 313318, 2017

(3) Naoki Abe, Shota Matsuhashi, Ryoji Onodera, Michiaki Shishido : "Development and evaluation of interface using masseter muscle potential and head posture" [in Japanese], Society for Science and Technology, Vol. 6, No. 1, pp. 35-40, 2017

(4) Grasso R, Glasauer S, Takei Y, Berthoz A : "The predictive brain : anticipatory control of head direction for the steering of locomotion", NeuroReport, Vol. 7, No. 6, pp. 1170-1174, 1996

(5) Eishi Hirasaki : "Head-eye Coordination during Circular Locomotion" [in Janapese], Academic Journal of Society of Biomechanisms Japan, Vol. 28, No. 1, pp. 3440, 2004

(6) Real Estate Fair Trade Council of Federation : "Implementation Rules for Fair Competition Regulations on Display of Real Estate" [in Japanese], http://www.rftc.jp/kiyak/hyouji_sekou.html\#001 (accessed 2018-11-16)

(7) Yasushi MATOBA and Satoshi SUGAYA : "Image Information Monitor System of Backward and High View for Wheelchairs" [in Japanese], Activity Report of University, Institute of Technologists, No. 1, pp. 8-13, 2010 